Canadian University Music Review

Revue de musique des universités canadiennes

\title{
Petrouchka : unité et dualité
}

\section{François Decarsin}

Numéro 6, 1985

URI : https://id.erudit.org/iderudit/1014035ar

DOI : https://doi.org/10.7202/1014035ar

Aller au sommaire du numéro

\section{Éditeur(s)}

Canadian University Music Society / Société de musique des universités canadiennes

\section{ISSN}

0710-0353 (imprimé)

2291-2436 (numérique)

Découvrir la revue

\section{Citer cet article}

Decarsin, F. (1985). Petrouchka : unité et dualité. Canadian University Music Review / Revue de musique des universités canadiennes, (6), 161-185.

https://doi.org/10.7202/1014035ar

(c) Canadian University Music Society / Société de musique des universités canadiennes, 1985
Ce document est protégé par la loi sur le droit d'auteur. L'utilisation des services d'Érudit (y compris la reproduction) est assujettie à sa politique d'utilisation que vous pouvez consulter en ligne.

https://apropos.erudit.org/fr/usagers/politique-dutilisation/ 


\title{
PETROUCHKA: UNITÉ ET DUALITÉ
}

\author{
François Decarsin
}

Bien que plus adaptée à la démonstration qu'à l'explication, l'analyse peut cependant faire surgir quelques hypothèses quant à la signification profonde de l'oeuvre si elle tient compte de tous les éléments disponibles. La lecture doit alors abandonner les hiérarchies habituelles (privilégiant les hauteurs) et adopter l'attitude préconisée par André Souris :

Une allusion aux mouvements internes des structures sonores, une interrogation sur la nature des forces de liaison interfonctionnelles, une recherche sur les mutations, les substitutions et les hiérarchies mouvantes des matériaux, enfin, ne fut-ce qu'une tentative de description des réseaux mobiles qui font de l'oeuvre musicale le siège d'une action foisonnante et polyvalente, que nous savons aujourd'hui irréductible, en fin de compte, à une définition uniquement rationnelle (Souris $1976: 268$ ).

Dans cette optique, une partition comme Petrouchka peut livrer quelques clés du langage de Stravinsky grâce à la liaison possible entre mécanismes propres à une oeuvre et procédés d'invention valables pour toute une trajectoire.

Stylistiquement, l'oeuvre de Stravinsky reste déterminée par une ambiguïté fondamentale déjà présente dans la phrase ellemême : absolument antihiérarchisée mais ordonnée, ultrathématique mais antidiscursive, violemment répétitive mais perpétuellement imprévisible. Plus généralement, le langage se caractérise par le refus de la tradition pour opérer une réduction romantique :

Loin de l'absorber jusqu'à l'exhaustion, il le rejette catégoriquement $(\ldots)$. Il demeure donc à un niveau plus élémentaire de l'investigation, ne tenant pratiquement aucun compte, en particulier, de la complexité formelle à laquelle le romantisme, en fin de course, avait abouti; loin d'assumer un héritage, il l'annihile (Boulez 1981 : 319). 
Hors l'héritage, il faut pourtant une syntaxe qui suffise au projet; celle-ci se traduira par une "pseudomorphose de la peinture " (Adorno) qui résulte de la suppression systématique des signes éventuels de raccordement entre les séquences. Paradoxalement, se trouve revalorisée une réelle précarité de l'instant musical, fortement liée à l'idée que se faisait Stravinsky sur le temps : " par l'imperfection de sa nature, l'homme est voué à subir l'écoulement du temps - de ses catégories de passé et d'avenir - sans jamais pouvoir rendre réelle, donc stable, celle de présent " (Stravinsky 1962 : 63). L'exclusion de la stabilité au profit d'une perpétuelle gravitation remplace les déclarations définitives de thèmes par une coexistence d'éclats sans qu'une dialectique puisse se dégager sur le plan technique ; car le résultat final de l'affrontement entre cellules - moteur essentiel de la phrase stravinskienne - n'est pas une synthèse mais l'expression figée de la dualité qui enveloppe l'énoncé en général. Et, malgré quelques points de fusion privilégiés (sensibles dans Petrouchka), les faisceaux ne se rencontrent pas mais c'est leur articulation complexe - n'excluant pas les interférences - qui produit l'unité globale.

Par ailleurs, la fusion totale entre la technique (l'écriture) et le style (le projet et ses racines) dessine le but ultime de la composition : " la manière de dire est la chose même (...) et, de ce fait, elle est toujours nouvelle" (Stravinsky 1958: 49). On pressent, à travers ces quelques formules laconiques, l'hostilité vis-à-vis des schémas normatifs, le refus de l'intégration stylistique pour une investigation (affranchie) du champ culturel disponible ; en découle alors cette technique de l'appropriation, du démontage et remontage de l'objet trouvé qui, loin de nier la culture, affirme au contraire la maîtrise de ses mécanismes. Cohérence du langage et rigueur du processus de composition résident presque exclusivement dans la perfection de l'intégration dans un contexte, selon une relation réellement organique dont Petrouchka produit un exemple quasi emblématique.

$\mathrm{Du}$ reste, la position stratégique de cette partition dans la production chorégraphique de 1910-1913 renforce cette relation en l'expliquant partiellement. 


\section{TABLEAU 1}

L'Oiseau de feu

Conte

Plans dramatiques juxtaposés

Individuel dominant sur le collectif

Séquences juxtaposées non regroupées

Emprunt au seul folklore russe

Chromatisme essentiel (diatonisme anecdotique)

L'individu : thème unique (Kastchéï et l'Oiseau)

Antagonismes rythmiques esquissés

Ostinato inexistant
Petrouchka

Histoire

2 plans dramatiques imbriqués

Équilibre individuel/ collectif

2 fois 2 tableaux: séquences regroupées

2 types de folklore

Diatonisme de base plus chromatisme dramatique

Thème double $(\mathrm{Pe}$ trouchka et le Maure)

Antagonismes rythmiques renforcés

Ostinato fréquent (pédales)
Le Sacre du printemps

Mythe

Pas de plans dramatiques " mise à plat "

Collectif dominant sur l'individuel

2 parties : séquences non regroupées

Rares emprunts au seul folklore russe

Diatonisme essentiel (chromatisme " vertical ")

Thème unique (l'Élue)

Antagonismes rythmiques décisifs

Ostinato essentiel (rythmes)

On voit qu'aux trois types de projets, un "conte dansé », des "scènes burlesques " et des " tableaux mythiques " correspondent trois courbes dramatiques donc trois énoncés musicaux différents. Avec l'Oiseau de feu, le ballet reste dans la lignée conventionnelle du narrativisme illustré, faisant naturellement alterner les séquences strictement scéniques (où l'action se noue et se défait par les gestes des personnages) et les séquences plus poétiques (peinture de la nature par exemple) indépendantes de l'action. Celle-ci, dans Petrouchka, se présente disloquée mais ses fragments sont organiquement reliés par une convergence aux niveaux de l'espace ("Fête populaire ") et du temps ("Scènes entre le Maure et Petrouchka ») : le ballet développe une histoire semi-réelle dans son principe, mais dont la chronologie reste évaluable avec précision. Les "Tableaux de la Russie païenne " situent le Sacre du printemps au niveau du mythe et non plus de l'histoire : la substance narrative reste évidemment sous-jacente, mais la réalité des personnages et de l'action s'est dérobée. Né d'une vision, le ballet met en relation des concepts et non des 
sujets : virginité, renouvellement de la nature, sont les principaux foyers d'une action mise à plat et non plus déroulée, et l'on pourrait même envisager une distribution différente des séquences sans altérer une chronologie qui n'est plus le mobile sensible de l'enchaînement. Les partitions elles-mêmes explicitent ces données : l'Oiseau de feu évite les actes mais renvoie au classique ballet à numéros, Petrouchka regroupe les scènes en deux fois deux tableaux tandis que le Sacre du printemps prend l'ultime recul avec ses deux parties ayant chacune un titre qui est un programme à lui seul. Le dernier clivage, qui découle évidemment des arguments, apparaît dans la répartition du collectif et de l'individuel. Ainsi, à la structure narrative de l'Oiseau de feu correspond une focalisation sur trois personnages principaux (l'Oiseau, Ivan, Kastcheï) qui donne matière à douze interventions solistes (ou en duo) alors que le groupe (les princesses) n'intervient que trois fois et la rêverie sous toute ses formes occupe six scènes. On perçoit la corrélation entre argument et texture dramatique en confrontant ce modèle à celui du Sacre : une seule irruption de soliste (l'Elue) si l'on excepte les six mesures du Sage ; et encore cette danse de l'Elue peut-elle apparaître comme récupérée par la majorité anonyme, mythique, indivisible :

En tant qu'individu, elle ne reflète rien que le réflexe inconscient et fortuit de la douleur : selon son organisation intime, son solo, comme toute les autres danses, c'est une danse collective, une ronde, vide de toute dialectique du général et du particulier (Adorno 1962 : 165).

Entre ces deux extrêmes s'inscrit l'équilibre complexe de Petrouchka au sein duquel le contexte poétique est le groupe même tandis que la séparation individu/groupe oscille entre le structurel (tableaux deux et trois) et l'anecdotique (tableaux un et quatre) : l'individu est sans cesse guetté par l'anonymat, bien qu'il soit thématiquement très typé et la foule se dissipe sans peine pour donner la parole aux isolés...

Plus généralement, la partition de Petrouchka reste le lieu privilégié de la rencontre aiguë des composantes les plus fortes du langage de Stravinsky : écriture de la dualité et dualité d'invention, qu'on isolera provisoirement pour mieux cerner leur convergence.

\section{La dualité comme ressort de l'articulation}

Interférences collectif/individuel

Premier lieu d'affrontement entre disparité et homogénéité, le 
traitement du collectif et de l'individuel reste évidemment lié au découpage des tableaux proprement dits, mais se retrouve aussi aux différents niveaux internes de l'oeuvre. Ainsi l'alternance, selon les tableaux, entre juxtaposition simple et imbrication subtile des motifs - caractéristique de toute la partition - n'est pas irréductible : les deux principes peuvent interférer dans une même séquence comme c'est le cas au premier tableau mettant en scène justement la multiplicité. Car la foule de Stravinsky ressemble à celle de Moussorgsky (scène "Dans la forêt de Kromy » de Boris Godounov par exemple) parce qu'elle se dissipe toujours à point nommé pour laisser parler les minorités, les isolés et trouer l'anonymat par quelques irruptions de motifs typés, ritournelles ou chansons plus importantes. Mais cette diversification peut ne pas altérer l'unité de l'ensemble et conserver quelques rapports (à décrypter) entre le collectif, l'impersonnel, et ses fuites ponctuelles.

Le début du premier tableau s'appuie essentiellement sur deux idées thématiques :

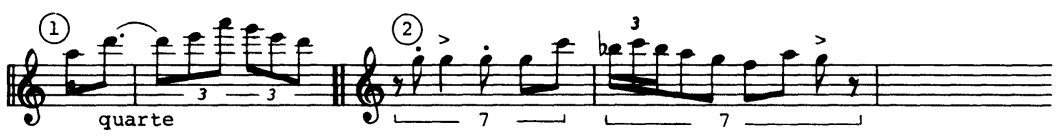

Exemple 1

L'épisode central de la scène (du chiffre 18 au chiffre 30) introduit plusieurs "corps étrangers", et notamment deux ritournelles fortement personnalisées, désanonymées, par la dislocation combinée de leur texture et de leur profil rythmique. Mais ce nouveau dipôle ne s'oppose pas radicalement à ce qui a précédé ; paradoxalement, il souligne progressivement l'unité de l'ensemble par quelques connexions remarquables bien que souterraines... Plus encore que l'anacrouse commune au premier thème et à la première chanson :

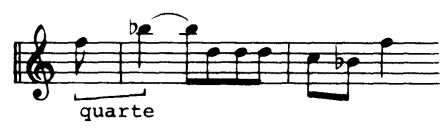

Exemple 2

(que l'enchaînement immédiat $25-26$ souligne ironiquement), c'est la parenté entre le deuxième thème et la deuxième chanson qui s'impose progressivement et irréfutablement : 


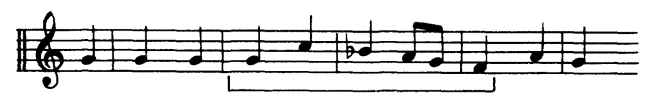

Exemple 3

Là encore, l'accent est mis sur la similitude par la superposition d'un signal propre au thème et de la fin de la chanson (deux mesures après 25). Il n'est pas indispensable d'enquêter sur l'aspect conscient de cette parenté, mais l'intérêt est dans la présence explicitée qui assure (en partie) l'unité de la scène: Schoenberg avouait bien un rapport de dérivation inconscient entre les deux idées principales de sa Symphonie de chambre op. $9 . .$.

Deux coïncidences sont peut-être plus troublantes qu'une seule : on relève la même propriété dans la " Danse des nourrices » où la coexistence des deux thèmes principaux concourt à l'unification ; après avoir été énoncés et développés successivement, ils laissent apparaître progressivement leur similitude (partielle mais évidente) par un cheminement commun, basé sur une même périodicité, une simultanéité des repères et des ruptures :

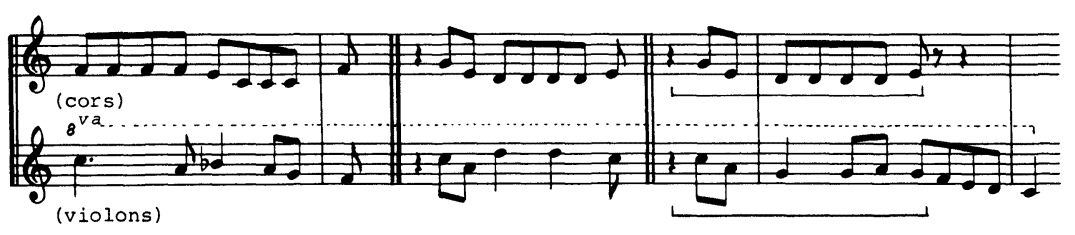

Exemple 4

Un deuxième lieu de conjonction entre la foule et l'individu est dans le traitement des quatre tableaux. Dépassant leur simple identité structurelle, les tableaux II et III (individus) renvoient aux tableaux I et IV (foule), en particulier au niveau de l'alternance entre ce qui peut être considéré comme thème et ce qui ne peut l'être. Soit, dans les peintures de foule, ce qu'on appellera "flou » (amalgame de pédales mélodiques, harmoniques ou rythmiques, l'immense " accordéon " d'Adorno) et, dans les séquences individuelles, le " non-thème " systématiquement illustré par un objet musical rapporté (valse, chanson...) ou par la cadence, segment de structure assez lâche inséré dans un contexte auquel il ne s'apparente pas forcément. Nous prendrons d'abord les deux épisodes se rapportant aux héros proprements dits. 


\section{TABLEAU 2}

Ile tableau : Petrouchka

a

introduction (93-95)*

$b$

A cadence (95-100)

C

thème (100-102)

$\alpha$

chansons + cadence (102-108)

B

$\beta$

danse (108-112)

$A^{\prime}\left[\begin{array}{c}b \\ \text { cadence }(112-116) \\ c \\ \text { thème (116-118) } \\ a \\ \text { introduction (118-119) }\end{array}\right.$
IIIe tableau : le Maure

a

introduction (120-121)

b

A

cadence (121-124)

C

thèmes 1 et 2 (124-134)

intervention 1 :

ballerine (134-140)

$\alpha$

valse $1(140-143)$

$\beta$

valse $2+$ thème 1 (143-148)

intervention 2 :

Petrouchka

(148-149)

$\alpha$

valse $1+$ thème $2(149-151)$

C suspension sur le thème de

Petrouchka (151-158)

*Les numéros renvoient aux chiffres de la partition.

La première particularité à relever est le recul - jusqu'à l'absence - des thèmes tels que les tableaux extrêmes nous les présentaient : airs de danses ou chansons typiquement russes, éléments imprégnés de folklore (répétitions, rythmes etc.). Ne subsistent que des motifs aux contours indécis : celui, ambigu, de Petrouchka (sur lequel nous reviendrons) et ceux, plus fluctuants encore, du Maure :

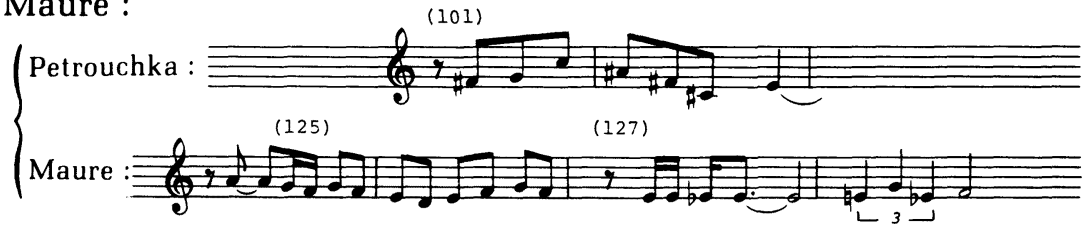

Exemple 5 
La deuxième est dans le système de développement lui-même : malgré le cloisonnement formel, l'enchaînement des idées se fait plus par tressage des motifs, ou superposition complexe, que par simple juxtaposition, car les " corps étrangers " (valse, chansons) ne se dissocient pas systématiquement des éléments propres persistant simultanément. Signe évident de l'adéquation à l'argument, un tel geste n'appartient qu'à ces deux tableaux seulement : Petrouchka (103) (chanson - cadence - chanson + cadence)/le Maure (144) (valse + thème), alors que les tableaux collectifs ne proposent, en regard, qu'une juxtaposition évidente, soulignée par les changements de titres (danses successives du $I^{\mathrm{e}}$ ) et plus encore par le profil excessivement marqué de chaque thème (comme nous le verrons).

La troisième particularité est justement cette présence de la cadence - élément strictement individuel - en même temps qu'un certain recul du pittoresque (sauf les deux motifs de Maure qui restent assez directement suggestifs).

Ce qui, par contre, distingue fondamentalement ces deux tableaux est leur articulation : concentration extrême du tableau Petrouchka sur un instrument privilégié, un thème unique, une architecture extrêmement raffinée, correspondant à l'ambiguïté du héros (forme repliée abc/ $\alpha \beta /$ bca) contre une enveloppe plus " ouverte " pour le tableau du Maure, ABC sans retour, suspensif, un orchestre diversifié plus présent (et mobile) et deux thèmes assez typés.

Mais, au delà de ces quelques particularités, l'unité l'emporte sur la divergence, car la récupération de l'individuel par le collectif vient de la nature même du matériau : sur le motif personnel, territorialisant, se greffe la chanson, la danse, que n'importe qui peut fredonner; après avoir signifié quelques individualités (premier tableau) au milieu de la foule, la ritournelle semble cette fois déposséder le personnage par l'éloignement aussi accentué que possible (mais lié à l'argument) entre les motifs du Maure, l'atmosphère créée par le timbre (126 en particulier) et les deux objets interrompant sa quiétude : solo de trompette, sorte de négatif de l'air de flûte du montreur de marionnette au premier tableau (135) et valse (140); ou encore par le contraste cru entre la danse martelée (108) faisant irruption au milieu des guirlandes ciselées suggérant la cellule de Petrouchka. Sans doute la dépossession est-elle beaucoup plus ressentie dans ce cas que dans celui où un isolé prend la parole au sein du groupe : reste alors à s'interroger sur la dimension que peut revêtir l'alternance entre 
thème et non-thème dans les peintures de foule, et l'effet de récupération au profit d'un traitement individualisant. On saisira alors la revalorisation du flou - ou du silence - comme élément actif dès que disparaît la cadence libre ou la chanson simple et on retrouvera les affinités entre attitudes a priori éloignées, comme résorption des contraires dans une unité organique poussée.

La conjonction entre peinture de l'individu et peinture du collectif est encore plus frappante dans les moments où thème et "flou » alternent simplement, immédiatement : refusant le silence, le déroulement devient alors oscillation entre le perceptible analy tique (un thème) et le perceptible statistique (la trame). Il n'est pas indispensable de détailler tous les cas, mais on ne peut omettre celui où thème, "flou " et cadence sont en relation étroite ; si les deux premières composantes sont typiques des tableaux I et IV et particulièrement du tableau $I V$, la vraie réunion des trois ne se produit qu'à la fin de l'oeuvre, et logiquement : le duel et la mort qui ont lieu en pleine place publique, inscrivent l'élément cadentiel, éclaté, au sein même de l'enveloppe collective fort discrète mais toujours en contact avec le drame comme le souligne le ralentissement général du mouvement interne à 263 (passage des lignes en double croches aux croches). Une telle fusion au niveau structurel pourrait bien renvoyer à la résolution d'un conflit plus profond, latent, au terme duquel l'isolé, le marginal, ne saurait connaître d'autre sort que son retour définitif au sein du groupe : le problème n'est alors plus essentiellement musical mais se pose au stade des motivations profondes, du choix et de la mise en forme de l'argument chorégraphique... Néanmoins, l'important demeure que la dissociation des courbes musicales spécifiques aux héros et à la foule (à plusieurs niveaux de distanciation réciproque) n'exclut pas la convergence. Les courbes individuelles sont typées : ambiguïté presque chaotique (II ${ }^{e}$ tableau), où la complexité du langage reproduit celle du sujet, en favorisant les fuites, hors des fonctions descriptives simples, de l'immédiateté ; conjonction plus nette entre langage et argument (III ${ }^{e}$ tableau), avec un recul du chaotique au profit du pittoresque, une réduction sensible des évasions au profit de la citation réinsérée, presque banalisée; dans les deux cas, absence du troisième personnage comme entité (la ballerine) mais apparitions précises dans le temps (objets musicaux cités). Parallèlement les courbes collectives décrivent le piétinement de l'action selon deux modalités : imbrications complexes de motifs et de citations (I ${ }^{\mathrm{er}}$ tableau) ou suite de danses (IVe) en favorisant systématiquement le timbre 
(pédale orchestrale comme composante facilement neutralisante) au détriment de la hauteur (thèmes simples, immédiats, absence de cadences). Mais les interférences masquées, souterraines, à décrypter, révèlent bien d'autres symptômes d'une dualité profonde qui laissent entendre que si la négation de la tradition n'est pas totale, au niveau formel principalement, la constitution d'un langage extrêment personnel passe par une technique du développement essentiellement axée sur une combinatoire complexe de la répétition et de la différence. Ainsi le problème de la forme trouve-t-il sa solution dans plus d'une séquence collective, où l'alternance simple reste le ressort unique (IV $\mathrm{I}_{\text {tableau, épisode }}$ des bohêmiens : un thème interrompt deux foix la trame installée au début; I er tableau: alternance déjà évoquée). Mais la perpétuelle remise en question de l'imbrication des cellules thématiques pose différemment la question de l'unification des divergences : comment construire un thème, une phrase, en ne gardant comme point de départ que les éléments les plus limités ou - le plus souvent - un seul élément ? On peut réunir les éléments de réponse en impliquant des composantes comme le phrasé, le timbre, dans l'ensemble des données habituelles (hauteurs, durées), et en interrogeant le premier mode d'écriture qui s'impose lorsqu'on évoque la combinaison du même et du différent : la variation.

\section{Engendrement et unité}

Plusieurs facteurs peuvent contribuer à définir l'articulation type d'un schéma de variation et Beethoven, en faisant reculer, de façon peut-être inégalée, le seuil de la distinction entre connu (le thème donné) et nouveau (ses métamorphoses) a montré toute l'ambiguïté de la problématique: sa musique n'est qu'une interminable exploration de ce qu'il est possible de faire d'autre avec ce qui est donné. Dans une optique très apparentée, ce qui, pour Adorno, n'est que gravitation perpétuelle autour du provisoire peut aussi bien être une métamorphose ironique de l'insignifiant : ironique parce que le geste qui consiste à s'emparer du banal pour en proposer d'innombrables variantes toutes les plus inattendues les unes que les autres (et faire oublier la banalité) peut relever du jeu, d'une pulsion de vie irrépressible, de l'arrogance incisive. L'effet est alors caractéristique : au faux piétinement, une répétition voilée, bref une démultiplication de l'unité qui étale et rassemble les possibles. Ce peut être sous la forme d'une variation simple impliquant directement les compo- 
santes traditionnelles: la danse russe du premier tableau reproduit la forme rondo la plus élémentaire en intercalant entre chaque refrain un même "couplet " toujours varié. Celui-ci apparaît en deux séquences (66) :

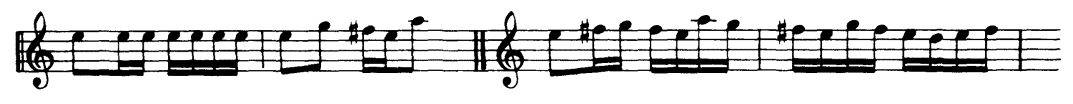

Exemple 6

où la deuxième semble stabiliser la première. Il réapparaît en introduisant un motif évidemment issu des précédents :

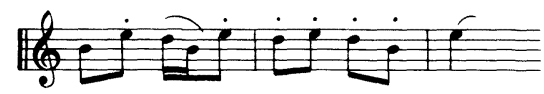

Exemple 7

et en développant ce motif par permutations et transpositions perpétuelles ; à l'intérieur de ce deuxième couplet se profile une série d'exposés différents par le timbre plus que par les hauteurs substituant à la différence explicite une différenciation plus subtile obtenue par la couleur ; ainsi l'émergence du chromatisme ascendant d'une apparition à l'autre du motif

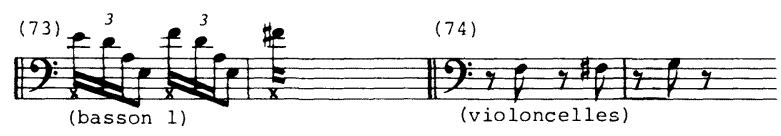

Exemple 8

efface la répétition sans supprimer l'identité : utilisé plus systématiquement dans Jeux de Debussy par exemple, un tel procédé revalorisera considérablement l'invention perpétuelle dans la cohésion.

On a déjà évoqué le mécanisme associant l'idée de thème à celle d'esquisse, selon lequel le définitif ne saurait exister sans être immuablement précédé par le geste qui l'a produit, la succession des coups d'essai. Ainsi la "Danse des cochers " s'articule-t-elle avant tout autour de la gestation apparente du motif unique, l'alternance admettant ici deux termes, et le silence. Chaque segment se présente comme un bloc dont seule une composante mobile laisse pressentir ce que sera le stade définitif : 

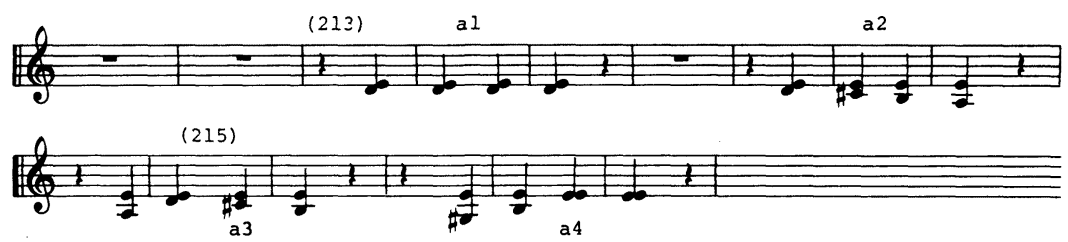

Exemple 9

et dessine ensuite exactement ce que sera le thème

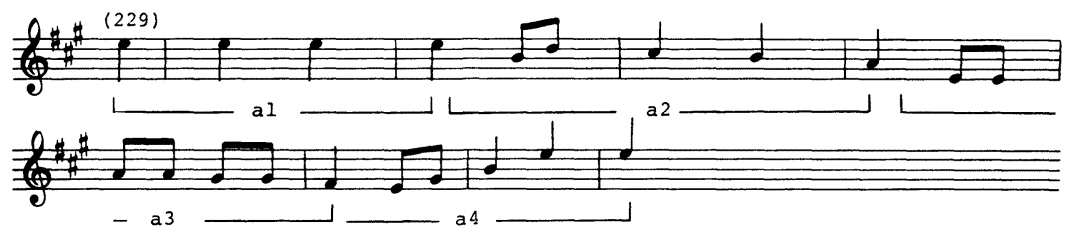

Exemple 10

tandis que les silences intercalaires invariables (2) facilitent la perception globale. La succession est la suivante: a $1 /|\mathrm{a} 2|$ \& a $3 \mid$ \& a4 - $2 d$ (remplissage du silence) - a2 - a 2 - $4 d$-rupture ( 5 mesures en syncope) - $4 d$ - a 2 - a3 - a 4 - $2 d-a 2$ - a4. Le remplissage du silence (les noires) puise dans le réservoir des notes du thème ; il est donc actif, participe effectivement au mouvement, à l'élaboration du rapport entre esquisse et finalité, dont relève directement le cheminement de l'idée thématique, et selon lequel l'écartèlement des éléments trouve toujours (progressivement) sa solution dans la synthèse, sans qu'il soit pour autant possible d'affirmer quelle forme préexiste réellement à l'autre : thème comme résultante ou comme point de départ d'un jeu...

Bien des exemples pourraient encore venir appuyer cette idée mais il en est un plus significatif encore (parce qu'impliquant une composante extrême discrète) qui éclaire parfaitement les propos précédents en témoignant du geste volontiers ironique qui caractérise si souvent l'invention de Stravinsky. Il est tiré de l'épisode des nourrices (IV tableau) et repose sur une simple transformation de l'accentuation. Bâti sur le motif suivant

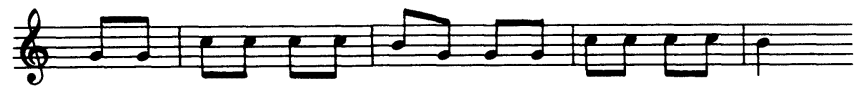


le déroulement propose quatre présentations qui définissent autant d'infléchissements du caractère de la danse : aimable, sophistiquée, narquoise, robuste... Bref, là encore, on peut se demander ce qui, de la ligne mélodique ou de tout l'orchestre, détermine l'autre; mais le plus important demeure la permanence d'un signal soulignant chaque modification dans les exposés successifs : doublure de l'accentuation par les violons et violoncelles, synchronisation d'une mélodie voltigeante avec les appoggiatures sur les temps forts, tassements du timbre et stabilisation générale de l'accentuation pour les piqués, enfin contretemps soulignés par tout l'orchestre dans le dernier exposé. Le simple fait que la relation entre les modifications discrètes d'une séquence strictement linéaire et l'enveloppe sonore globale soit si évidente réaffirme le souci de ménager constamment la différence au sein d'une structure sans engager l'homogénéité, l'unité de celle-ci : la répétition - puisqu'elle domine largement ici - gomme les différences mais ne vit réellement que par elles...

Aux niveaux harmonique et thématique, la répétition décentrée d'un élément simple peut également engendrer un type de développement autonome, si complet en lui-même qu'il peut conclure à lui seul une partie. Ainsi la dernière séquence de la danse russe, sorte de coda scintillante, faussement mobile, repose sur deux types d'alternance : rythmique, avec l'opposition de deux cellules $\mathrm{a} / \mathrm{b}$, et harmonique, par l'oscillation tonique/dominante perpétuelle. La dualité du déroulement est d'abord interne,

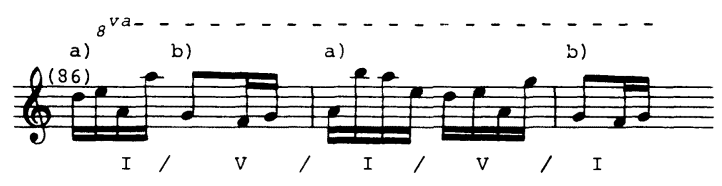

Exemple 12

parce que cette alternance linéaire éclipse l'enveloppe harmonique stable et discrète; elle est ensuite généralisée parce que l'indécision harmonique se hisse au premier plan et cumule avec les ruptures rythmiques précédentes :

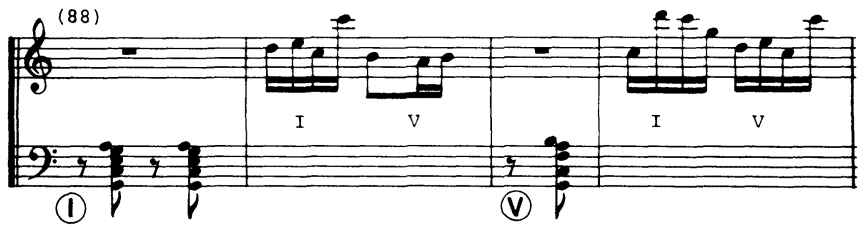

Exemple 13 
Ces deux séquences immédiatement reliées ne s'affrontent pas entre elles : elles ne font que présenter deux types d'antagonismes qui seront résolus à l'ultime moment; la cadence parfaite (masquée) rallie en un seul geste et les soubresauts rythmiques et l'ambiguïté harmonique qui ont prévalu.

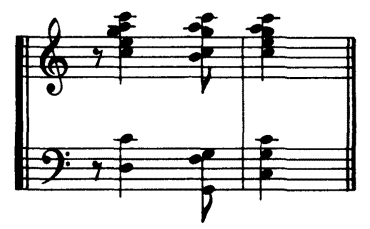

Exemple 14

Le même type de résorption des antagonismes peut être actif au niveau proprement formel comme en témoigne l'affrontement des deux motifs à la fin de l'oeuvre (chiffre 240). Le premier est mobile

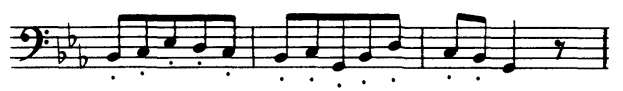

Exemple 15

le second ne le devient qu'à sa troisième apparition pour s'imposer apparemment (242). Or c'est le premier qui s'installera définitivement en passant du stade rythmique pur à la mélodie (243). Cette mélodie rappelle d'ailleurs le grand thème russe du premier tableau en mêlant à sa courbe relativement simple un contrechant syncopé sur une seule note :

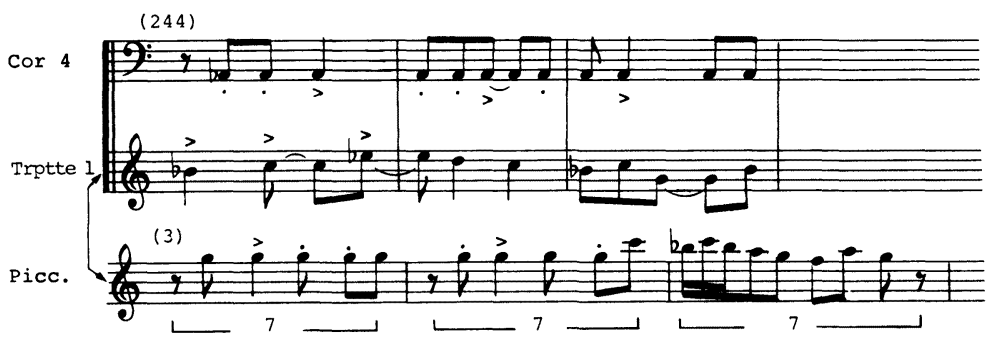

Exemple 16

Ce dernier thème se dessine donc logiquement comme un commentaire de la ligne du précédent 


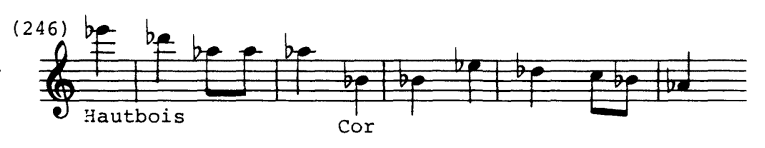

Exemple 17

et en le raccordant à la triade mélodique commune aux deux, laquelle devient progressivement la triade harmonique conclusion de l'oeuvre (avec un ordre des degrés permuté : V-IV-I).

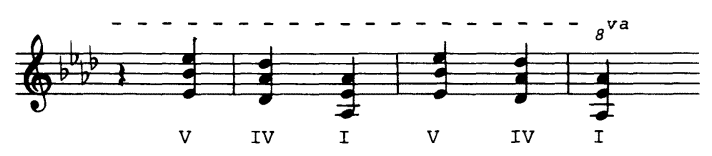

Exemple 18

On voit que, même dans le cas de dérobement d'une idée devant une autre, il y a toujours un terme sous-jacent dont la principale propriété est de résoudre ce qui a priori semble inconciliable, tout en différant l'unification par une errance que la virtuosité ne se lasse pas d'entretenir et de compliquer...

On ne saurait conclure cette approche des fonctions unificatrices de l'écriture de Petrouchka sans réserver quelques lignes à l'étude du héros lui-même dans ses deux apparitions les plus remarquables, et symétriques sur le plan dramatique comme thématique. On peut les isoler cependant et considérer l'élaboration du thème de Petrouchka telle qu'elle est entièrement écrite au chiffre 101. La relation entre l'ambiguïté de la courbe thématique - ascendante puis descendante (avec un point d'arrivée plus bas que le point de départ, comme une ligne de défaite) - et celle de la forme du tableau tout entier est frappante: que la partie descendante du thème succède à une cadence plutôt ascendante du piano dans la première partie (100) pour devenir l'inverse à la fin de la troisième partie (116) corrobore la duplicité inhérente au profil même du thème ; et si la conjonction harmonique fameuse (do/fa dièse) surgit comme le résultat fortuit d'un jeu spontané sur les touches blanches et les touches noires du clavier, elle renvoie pourtant à une réflexion consciente sur le problème de l'élargissement de la tonalité (sans l'abandon) comme en témoigne le contexte même du thème : la simultanéité do/fa dièse est rendue par la vitesse, 


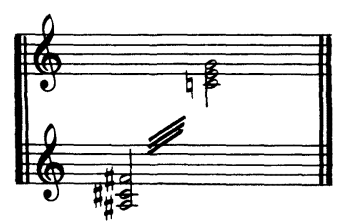

Exemple 19

avant que ne se déploient successivement les deux composantes harmoniques

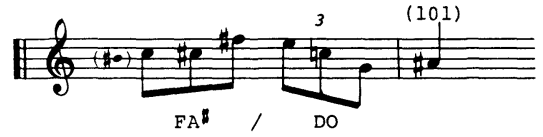

Exemple 20

Cette dualité thématiques est évidemment celle du héros : vindicatif mais en infériorité, claironnant mais fragile, comme l'arpège auquel il s'identifie, ressort essentiel au duel final où Petrouchka règle seul son propre sort, tandis que toute la dualité de l'oeuvre se résout dans un bref affrontement entre les deux moitiés du même personnage.

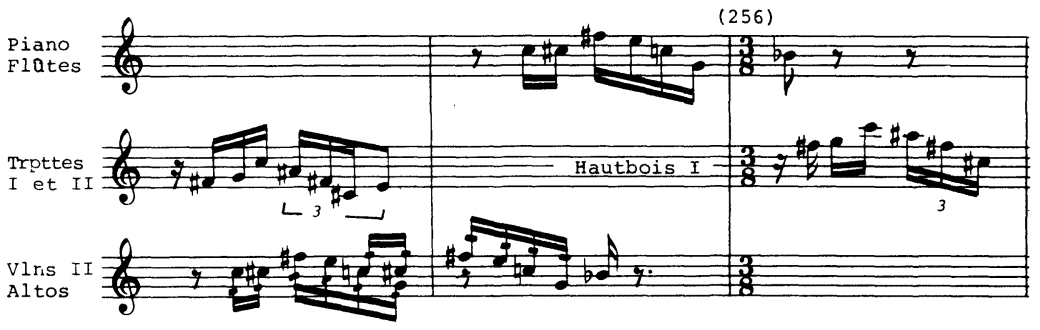

Exemple 21

Au delà de ce moment, tous les motifs réapparaissent figés, immobilisés ou dépersonnalisés, dans une accélération étrange, un brassage des éléments les plus disparates (motif en canon de Petrouchka, chromatisme descendant évoquant le Maure, rappel de la naissance des trois marionnettes...) qui jouent un dernier tour à l'unité indissoluble de la trajectoire de Petrouchka. Et l'apparition finale du double au dessus de la scène - qui reste pour Stravinsky le vrai Petrouchka, l'autre n'étant qu'une marionnette 
- scelle l'unité de l'oeuvre d'une façon... bien ambiguë : "Le fantôme à la fin est le vrai Petrouchka... le personnage de la pièce (précédente) seulement une poupée... Le geste du " fantôme "... n'est pas tant un signe de triomphe qu'un pied de nez au magicien " (Craft and Stravinsky $1979: 67$ ).

\section{Dialectique objet trouvé/objet créé}

\section{Dans Petrouchka}

Ce second aspect de la problématique définie par Petrouchka - coexistence de deux types d'inspiration dans une seule élaboration musicale - appelle une réflexion sur les sources mêmes de l'invention stravinskienne, comme somme d'un certain nombre de facteurs plus ou moins avoués mais toujours agissants. Car il est évident que le regard jeté sur les éléments culturels disponibles dépasse systématiquement le simple mécanisme inspiration/citation pour s'élever à une dimension supérieure, celle justement où il devient difficile de définir la part réelle du modèle et le degré de sa transformation. On saisit mieux ce que cette tentative de distinction a d'aléatoire quand on lit, sous la plume de Stravinsky, qu'un seul motif des Noces est d'origine populaire et que tous les autres sont réinventés.

Il n'entrait pas dans mon intention de reconstituer les rites des noces paysannes, et les questions ethnographiques me préoccupaient fort peut $(\ldots)$ Je m'inspirais de ces coutumes mais tout en me réservant la liberté complète d'en user comme il me conviendrait (Stravinsky $1967: 116$ ).

Cette duplicité est beaucoup plus facile à décrypter dans Petrouchka ou dans Mavra (qui contiennent des pans entiers de musique rapportée) que dans d'autres oeuvres - comme par exemple l'Octuor à vents - qui intègrent des modèles essentiellement formels et surtout, selon les mots de Boucourechliev, " une typologie d'écriture caractéristique de ces formes, virtuosement développée : mais sans la signature d'une époque ou d'un style suffisamment déterminés " (Boucourechliev 1982: 204). Petrouchka appartient à cette période où les oeuvres transgressent l'emprise du moment et non à celle où, selon Schaeffner, « ... ces éléments réunis par le compositeur ne sont point d'exception et revêtent un caractère d'universalité, sinon d'impersonnalité " (cité dans Boucourechliev 1982 : 204). En outre, la mémoire (remarquable) favorise l'irruption des éléments les plus hétéroclites, comme remise à jour des acquisitions les plus récentes (rengaines 
entendues à Beaulieu-sur-mer, ou valses de Lanner entendues partout). Mémoire et culture se confondront ailleurs pour produire une polka ou un choral (Histoire du soldat) mais elles donnent ici déjà quelques épisodes où sont brassés le connu et l'inventé.

Car l'inspiration l'emporte systématiquement sur la réflexion, la prédétermination; aussi l'absence d'une rhétorique expressive avouée n'est-elle pas

l'effet d'un simple parti pris d'antiromantisme. Elle découle du projet fondamental qui est à l'origine de toutes les oeuvres du compositeur, et qui consiste à n'utiliser les matériaux du langage musical que dans leurs fonctions les plus essentielles en même temps que les plus concrètes (Souris $1976: 228$ ).

Stravinsky n'est pas un intellectuel - il l'a répété! - "et par conséquent les problèmes de l'explication ne sont pas d'un grand intérêt " (Stravinsky 1962) ; ceci n'empêche pas les résurgences apparemment spontanées, naturellement inscrites dans le déroulement musical, de relever toutes de ce projet fondamental déjà évoqué : éviter l'expression sur le mode $x$ ou y pour imposer l'archétype du domaine de l'émotion en question.

La description, l'évocation, perdent donc une grande part de leur sens avec l'emploi de la citation, qui fait du sujet un élément secondaire : "Lorsqu'il intervient en musique, il ne peut être que subsidiaire, soit qu'il coïncide avec le sens symbolique des formes soit qu'il s'y oppose, ou qu'il le complète " (Souris 1976 : 228). Le mode d'expression n'existe pas en soi car l'objet est le sens, par le jeu d'une totale distanciation ; ainsi l'entrée de la ballerine (135), comme le solo du montreur de marionnettes (60), restent strictement rudimentaires quant au timbre (un seul instrument), au rythme (simple au mètre (carrures symétriques) au profil mélodique sûr (antécédent/conséquent équilibrés et clairement dissociés) :

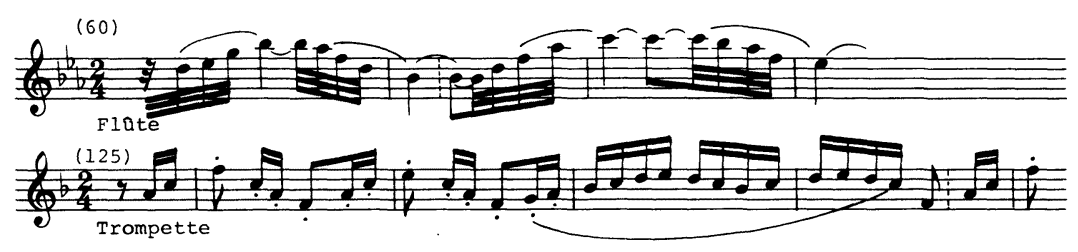

Exemple 22 
Mais la présence même de l'objet rapporté peut infléchir la perception d'une forme, par sa simple irruption qui remet en cause le rapport entre un tout et ses parties, au sens où les théoriciens de la forme ont défini ces paramètres. Si le musicien n'est pas censé appliquer consciemment une théorie définie dans son oeuvre, celle-ci peut pourtant être quelquefois le reflet d'une évolution de la pensée (comme celle de Debussy l'est par exemple de la notion de durée bergsonienne). Or un des corollaires de la Gestalt theorie est la reconsidération du couple forme/parties, contenant/contenu, dans la mesure où précisément une forme " est autre chose ou quelque chose de plus que la somme de ses parties" (Paul Guilleaume). Dès lors, les différentes résurgences de souvenirs musicaux deviennent plus qu'illustration immédiate, et on peut considérer les trois chansons citées dans le deuxième tableau comme autre chose que de simples passages au style direct pour leur conférer un rôle pratiquement sémantique ; ainsi des motifs comme ceux-ci :

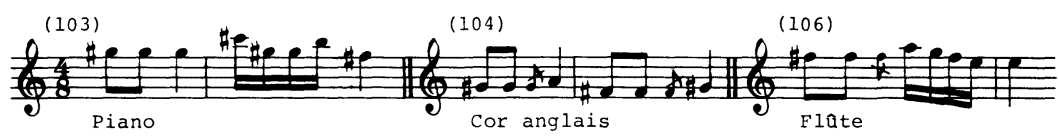

Exemple 23

qui n'ont, en eux-mêmes, aucune dimension particulière, en reçoivent une par leur fusion dans un contexte chaotique, éclaté, où ils deviennent des pôles de détente, des agents " territorialisants ". Leur fonction n'est que le résultat d'une interrelation avec d'autres paramètres (timbre, vitesse) dont la nature n'offre aucun point commun avec la leur. Il n'y a pas de dérivation évidente entre les éléments cadentiels précédents et ces chansons. Et on ne peut considérer l'enchaînement des uns aux autres comme une simple addition : la succession créé la forme ou du moins une de ses particularités, mais n'en résulte absolument pas. Ainsi, dans le troisième tableau, l'irruption des deux valses (empruntées à Lanner) constitue la digression, la distraction après la concentration : celle du Maure occupé à peler son orange, mais aussi celle des motifs tendus, apériodiques, faussement répétitifs. Là encore, les valses voient leur anonymat - et leur connotation sociologique modifiés, au service d'une relation en remettant en cause la notion de trajectoire formelle préétablie, et prolonge le modèle analytique de la théorie gestaltiste ; car comme l'a écrit Levi-Strauss, "le 
contenu... tira sa réalité de sa structure, et ce qu'on appelle forme est la mise en structure des structures locales, en quoi consiste le contenu " (cité in Boulez 1963 : 31). On pourra objecter que de semblables effets se retrouvent chez bien d'autres compositeurs ayant utilisé le collage (Ives, Cage, Kagel, Berio) ; mais contrairement à ceux-ci, Stravinsky ne place pas son but dans une juxtaposition de séquences différentes ; la justaposition accidentelle, ponctuelle, acquiert une propriété particulière échappant à la nature même de ses éléments: celle de faire bifurquer un déroulement musical somme toute traditionnel en lui ajoutant une dimension supplémentaire et variable, la distanciation, deuxième pôle de l'invention stravinskienne.

On connaît les métamorphoses des motifs folkloriques puisés dans le fonds russe - notamment le recueil de chants réunis par Rimsky Korsakov d'après Balakirev - qui vont de la simple permutation de notes (ou de fragments) à la reconstruction totale. Petrouchka accumule les citations du premier type depuis les cris des vendeurs de marrons jusqu'au " chant du Volochebniki » (311 à 358) en passant par le motif du "Petersky » contrepointé par une autre mélodie célèbre, "Akh vy sieni, moy sieni " (183) ainsi que l'a montré Irina Vershinina dans son étude sur les premiers ballets de Stravinsky (cité par Craft and Stravinsky 1979 :608-09). Dès le Sacre du printemps, le démontage devient plus systématique et engendre de nouvelles idées comme par exemple la phrase initiale du basson :

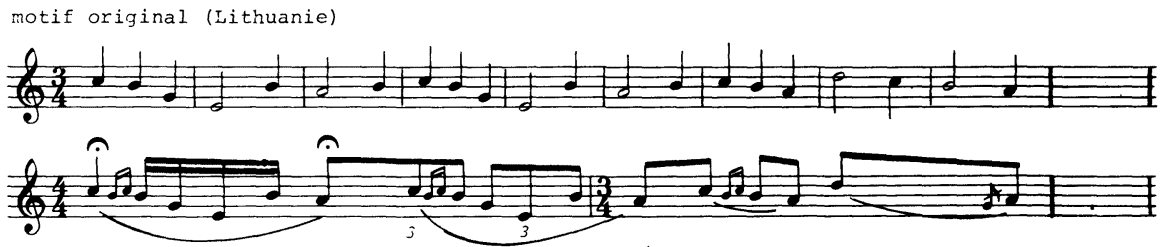

Exemple 24

On peut explorer interminablement les niveaux de transformation conduisant de la source à l'idée, mais le geste le plus remarquable (marquant toute la création de Stravinsky) est dans le mode de personnalisation de l'objet trouvé ; nouveau signe de cette dualité de la pensée déjà évoquée, celui-ci peut être interprété de deux façons : soit, appliqué dans Petrouchka, comme principe de distanciation (le simple déréglage du mécanisme ne fait que 
souligner le recul pris par rapport à l'objet choisi), soit comme greffe sur une structure absolument indépendante (cas le plus fréquent) dans une relation de succession, de simultanéité, ou de fusion.

Ainsi le travail effectué sur la première valse dans le troisième tableau appelle-t-il plusieurs remarques ; la formule d'accompagnement offre déjà une équivoque :

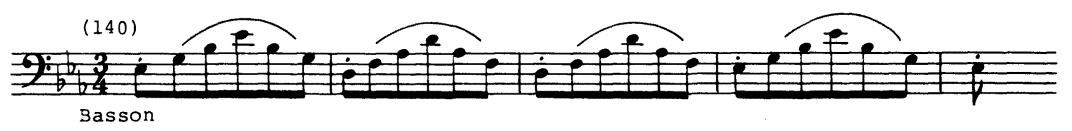

Exemple 25

Les six croches peuvent être perçues comme deux fois trois croches, soit deux mesures de valse à $3 / 8$ (ou une à $6 / 8$ ) à cause du phrasé, du tempo exagérément lent ; à cette déformation physique s'ajoute une entorse métrique au niveau de la mélodie qui ne coïncide pas exactement avec le schéma harmonique le plus attendu :

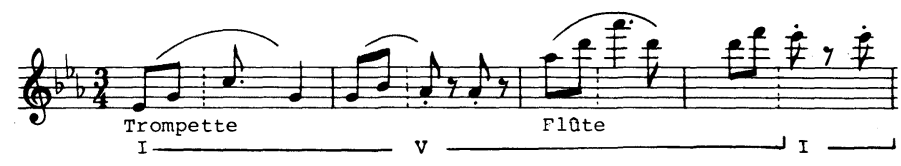

Exemple 26

Là encore, une accentuation particulière fractionne la phrase à son point le plus discret, et tend à légèrement fausser la perception d'une valse paisible, alors que tous les éléments sont réunis : timbre, vitesse, simplicité harmonique/mélodique... Peut-être ya-t-il corrélation entre ce traitement d'un schéma rebattu et la conception modernisée de l'automate scientifique développée par Xenakis :

Un automate se présente comme un réseau de causes et d'effets, c'est-à-dire une chaîne temporelle d'événements, couplée ou multicouplée $(\ldots)$ un automate peut être fermé. Il suffit de brancher l'énergie et il fonctionne cycliquement. Il peut être relativement ouvert avec des données d'entrées (...) et, malgré une rigidité interne qui définit l'automate, il peut produire des résultats différents chaque fois que l'on change les données d'entrée (Xenakis $1979: 97-98$ ). 
Précisément, la victoire remportée ici par un déroulement fantaisiste sur une syntaxe répétitive signifie la possibilité pour l'invention de modifier l'amorphe, l'objet inanimé, non pas seulement en le projetant arbitrairement dans un contexte étranger, mais en le dotant de nouveaux modes d'articulations qui, sans changer sa structure ni même sa finalité, en modifient les performances pour le rendre opérationnel à tout moment.

Incidences ultérieures

Une des plus remarquables manifestations de cette faculté est la coexistence entre sérialisme et " stravinskisme " après 1945. On a vu que les bases du langage s'étaient très vite établies à l'image du chant russe et que le diatonisme en était la composante la plus éminente au point que l'identification du seul chant russe réel des Noces reste problématique dans un réseau où toutes les autres idées sont semblables... mais inventées. L'interaction est là : les Noces "font " folklorique, les oeuvres sérielles "font " du Webern, mais toute la production est de Stravinsky, depuis la fêlure de notre valse jusqu'à l'orchestration des deux Lieder de Hugo Wolf. Et "faire du Webern", c'est avant tout inscrire le chromatisme comme ligne de force ; ainsi la série d'Agon se réfère aux Variations op. 30 par son profil mélodico-harmonique, avec la même pondération de l'accord majeur/mineur comme " différenciateur harmonique " (Boucourechliev).

Webern :

Variations, op. 30

Stravinksy :

Agon

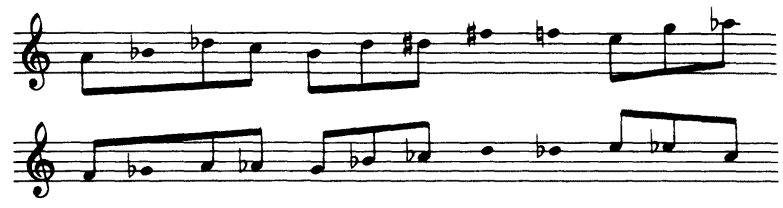

Exemple 27

Mais c'est le cas particulier que peuvent faire oublier d'autres motifs sériels qui eux donnent une place déterminante au diatonisme le plus évident :

Threni

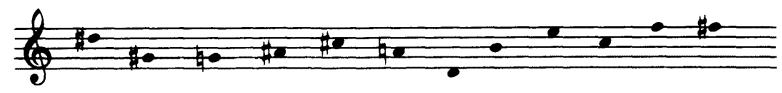

Epitaphium

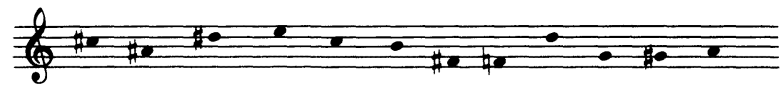

Exemple 28 
La raison :

Je n'ai assurément pas commencé (ce que je ne ferai jamais) par une idée purement sérielle et en fait, en commençant, je ne savais pas ni ne me souciais de savoir si les douze notes seraient toutes utilisées. Quand j'eus écrit la moitié à peu près de la première phrase, je vis cependant son dessin sériel et peut être alors commençai-je à travailler dans le sens de ce dessin (Craft 1963).

L'inspiration - qui n'est avant tout qu'un produit de la culture - parle avant toute élaboration ; en cela, elle fournit un matériau de base immuable, façonnable à volonté mais aux structures radicalement imposées. Comme la valse - ou le choral ou la marche - ont reçu un nouveau système d'articulation, le profil des différentes séries montre que les antécédents individuels ne sont jamais liquidés même si, consciemment, l'histoire est oubliée. Bien avant la série, cependant, quelques motifs ont porté une signature commune, dans une langue différente de celle qui l'ont précédée. Coïncidence? Ces motifs renvoient systématiquement à la peinture d'un individu :

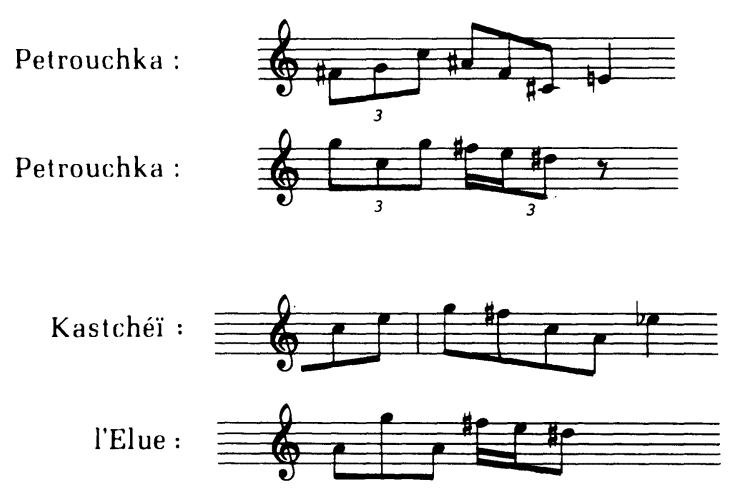

Exemple 29

Une telle unicité de pensée peut expliquer ou cautionner partiellement cette esthétique de la déformation, de la transformation. Il y a moins de distance qu'on ne pourrait l'imaginer entre la mutation d'un objet fort simple en ligne imprécise par seule opération sur la carrure, les points d'appui, et celle d'un pentacorde élémentaire dispersé de la façon la plus sophistiquée : 


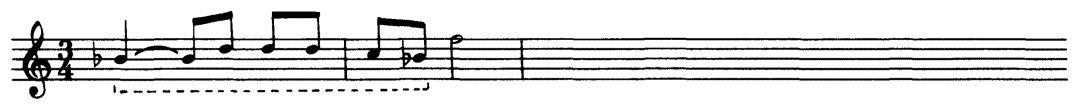

$1^{\text {er }}$ Chant d'après Shakespeare

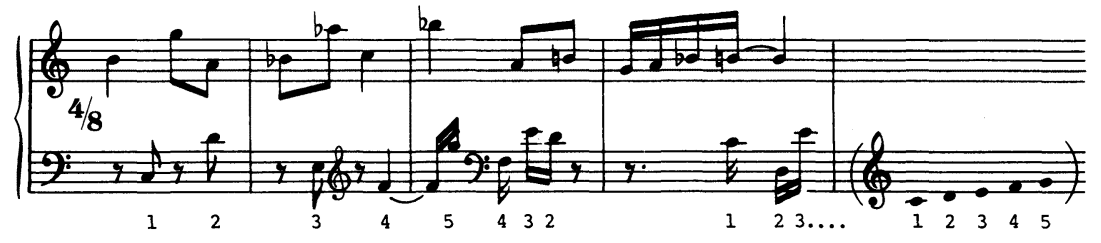

Exemple 30

Abolition de l'histoire ? Le terme est peut être un peu forcé. Mais il est certain que l'ensemble des styles de Stravinsky a gardé un centre de convergence, et a constamment tendu vers la définition de ce que Boucourechliev appelle un "espace " qui graviterait fondamentalement autour du timbre, composante structurelle et non illustrative. Une telle hypothèse vérifie deux attitudes fondamentales dans toute la trajectoire du compositeur. La première est le recours au collage, à la citation, à l'objet étranger, comme intégration dans un des courants artistiques les plus éminents de ce siècle, le cubisme, né d'un changement de regard sur l'objet, d'une reconsidération du rapport entre le détail et l'enveloppe formelle :

Voici que, tout à coup, un homme vient qui renverse tout. Il voit la pomme comme insaisissable et la montagne comme un objet maniable et déformable à volonté (...) Nous sommes dans une époque où ce qui change, ce sont les distances psychiques plus encore que les distances géométriques (Francastel $1970: 225$ ).

Ces lignes sur Cézanne pourraient s'appliquer à Stravinsky et pas seulement celui des ballets russes ! La deuxième est la permanence d'une pensée créatrice au delà des différents styles qu'elle a adoptés pour s'épanouir. Petrouchka renvoie à la dernière période du compositeur, celle où le langage personnel a résisté aux ultimes sollicitations, mais ne relègue pas automatiquement la succession des choix intermédiaires. En cela l'oeuvre resurgit naturellement comme le foyer extrêmement dense où s'élaborent - où sont dẹjà formulés parfois - les certitudes et les doutes, les errements et les choix, le délire et la rigueur. 


\section{REFERENCES}

ADORNO, T.W.

1962 : Philosophie de la nouvelle musique. Paris : Gallimard.

BOUCOURECHLIEV, A.

1982 : Stravinsky. Paris : Fayard.

BOULEZ, P.

1963 : Penser la musique aujourd'hui. Lausanne : Gonthier.

1966 : Relevés d'apprenti. Paris : Seuil.

1981 : Points de repère. Paris : Bourgois/Seuil.

CRAFT, R.

1963 : Souvenirs et commentaires. Paris : Gallimard.

FRANCASTEL, P.

1970 : Études de sociologie de l'art. Paris : Gonthier.

SOURIS, A.

1976 : Conditions de la musique et autres écrits. Paris/Bruxelles :

Édition du Centre National de la Recherche Scientifique./ éditions de l'université de Bruxelles.

STRAVINSKY, I.

1958 : Avec Stravinsky. Monaco : Éditions du Rocher.

1962 : Chroniques de ma vie. Paris : Denoël.

STRAVINSKY, V. and CRAFT, R.

1979 : Stravinsky in Pictures and Documents. London : Hutchinson. XENAKIS, I.

1979 : Arts/Sciences/Alliages. Paris : Casterman. 